\title{
Let the experts do the hard work for you
}

Innovative dental software developers, Welltime, are excited to announce their latest helpful solution: the Dental Diary app.

With this easy-to-use and free to download app, you will be able to access your entire diary on your smartphone - whenever you need to. Syncing with your practice's existing management software, the Dental Diary app will update automatically if appointments are made, changed or cancelled - allowing you to stay in complete control of your workload.

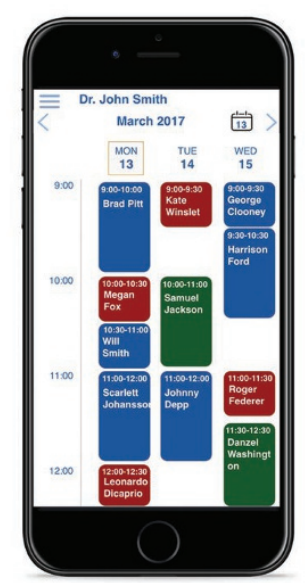

Featuring 24/7 access, Dental Diary will let you plan your time more effectively. Updating in real time, the app will let you know if your first appointment tomorrow has been cancelled, allowing you to plan your day quickly and simply. What's more, you'll also be able to access your team's diaries, too - meaning your entire practice will run more efficiently than ever.

The Dental Diary app from Welltime is available for download on Android and iPhone; take control of your diary and stay informed at all times. To find out more, contact the expert Welltime team today on 07999991 337, email sales@welltime.co.uk or visit the website at www.welltime.co.uk.
Do you ever feel like you've got enough to deal with every day, without having to worry about the maintenance of your valuable handpieces or unexpected handpiece repair bills? If your answer is 'yes', then you'll love Trigiene's new 'Handpiece Service Care Plan', offering a choice of flexible handpiece maintenance and repair options, especially designed to suit your individual needs and budget.

Starting from as little as $£ 8.99$ a month, your handpieces will be fully protected with regular scheduled maintenance, testing and key repairs ${ }^{\star}$ by Trigiene's qualified service personnel. Trigiene's dedicated team will ensure complete equipment reliability, whilst helping to identify any potential issues before they arise. Trigiene's Service Care Plan gives you compliance assurance of annual servicing and repairs as well as an advanced online tracking and reporting system.
Trigiene Dental understands that your handpieces are some of the most important pieces of equipment in your practice. Aware of how much dental professionals rely on their handpieces, Trigiene strives to give you total peace of mind against the risk of downtime and unexpected repair costs. They promise to service, maintain and repair all major brands of handpieces, extending the life of your equipment, whilst ensuring maximum performance to keep your practice running smoothly.

With a Trigiene Service Care Plan our experts do all the hard work for you, maintaining your handpieces so you don't have to. For more information call Trigiene's service team on 01642442910 or visit www.dentalhandpiecerepairs. co.uk.

*Terms and conditions apply, includes premium quality parts and ceramic bearings for all turbines.

\section{Any company can make an intraoral scanner...}

...Making it fast, accurate, easy and open is what Carestream Dental does.

The CS 3600 intraoral scanner from Carestream Dental makes it easy for you to elevate your practice standards and distinguish yourself from others.

Its high speed continuous scanning delivers a super fast, simple, smooth and efficient user experience. Additional features such as the intelligent matching system and scan data history also enable you to complete missing data at any time and remove excess scanned tissue for a more refined digital impression.

Precise, 3D HD colour images are produced every time, which can be sent to your laboratory of choice as open.STL files in a matter of minutes.

Indicated for use in restorative, orthodontic and implant-borne restorative dentistry, the CS 3600 is the perfect chairside partner for every practitioner.

To find out more, contact the friendly team at Carestream Dental today on 0800169 9692 or visit www.carestreamdental.co.uk.

\section{Revive your business}

'Forgotten' or unsold treatment plans could be costing your practice between $£ 100,000$ and $£ 1.3$ million. Effectively reviving them could therefore be a highly lucrative activity and now there's an easy way to do it.

Working with the experts from 7 connections, you can make use of their series of reactivation emails designed to re-engage patients who have shown interest in particular treatments in the past. The team will help you communicate the right messages to the right people at the right time, enabling you to strengthen your patient relationships and ensure they come to you first when they are ready to proceed with treatment.

What's more, the service is fully automated, so you can focus on your patients in the practice and deliver the highest standards of care, while the 7connections Revive emails do your marketing for you.

One dentist made $£ 38,000$ in extra sales within a month of using the automated reactivation sequences from 7 connections.

For more information about 7connections, call 02030957259 or email hello@7connections.com. www.7connections.com. 